\title{
HARMONIC ANALYSIS OF A SOURCE IDENTIFICATION PROBLEM
}

\author{
BY \\ E. M. ARNOLD \\ Battelle, Pacific Northwest Laboratory
}

1. Introduction. The object of this note is the problem of identifying a source in a porous medium which hosts a steady flow field. The medium properties and the velocity potential are assumed known. At first the known functions will be considered as deterministic. Later the stochastic source identification problem will be considered. Thus, we seek a function $f$ satisfying:

$$
\operatorname{div}(K \operatorname{grad} \phi)=f
$$

on a region $D \subset \mathbb{R}^{N}$ with known potential $\phi$ and permeability $K$.

In the physical situation, $K$ is determined in a laboratory test of a core sample and can be quite unrepresentative of the real permeability. The potential is more accurately measured as the height of a water column, but is also subject to some measurement error. Consequently, a direct calculation of $f$ by performing the differentiations on the left of (1) could lead to large errors. The alternative method presented here is different from recent source identification methods used by Cannon and DuChateau [1] for parabolic equations in that hard-to-obtain interior data is used. On the other hand, our method treats more general kinds of source terms and leads to an explicit integral formula for the solution. This formula is used to show that the source $f$ depends continuously on $K$ (or $\phi$ ) in the $L^{p}$ sense. This continuous dependence is based on a Fourier multiplier theorem and $L^{p}$ estimates of spherical maximal functions. It has little in common with the method of searching for a solution to an inverse problem in a compact subspace of naturally occurring functions, and/or using a regularization method (cf. Tikhonov [2]).

The integral formula for $f$ can be extended to represent the solution $f$ of a corresponding random source identification problem. In this setup $K, \phi$, and $f$ are defined on $D \times \Omega$ where $\Omega$ is a sample space with an associated probability measure. Statistical estimation techniques can be used to interpolate the given data for $\phi$ and $K$ in $D$ and to generate pointwise means and variances. These statistical quantities can then be used with the results of this note to give means and estimates of the variances at each point of $D$ for the function $f(x, \omega)$. This is a major justification of the method.

2. Source identification on $\mathbb{R}^{N}$. First the case $D=\mathbb{R}^{N}$ will be considered. This assumption allows the use of Fourier transforms and convolutions of functions and measures. In the following section the analyses will be extended to treat bounded domains

\footnotetext{
* Received November 10, 1981.
} 
$D$. In order to be able to operate on a formal level it will be temporarily assumed that $K$, $\phi$, and $f$ are in $C_{c}^{\infty}\left(\mathbb{R}^{N}\right)$, the space of infinitely differentiable functions with compact support.

If both sides of (1) are integrated over the unit ball $B(x)$ at $x$, with boundary $S(x)$, Green's theorem yields:

$$
\int_{S(x)} K \frac{\partial \phi}{\partial n} d s=\int_{B(x)} f d y
$$

where $d s=$ surface measure and $d y$ is Lebesgue measure on $\mathbb{R}^{N}$ and $n=\left(n_{1}, \ldots, n_{N}\right)$ is the outer unit normal. Since $K$ and $\phi$ are assumed known, the left side of (2) is a known function of $x$ denoted by $A(x)$. Later, when continuous dependence results are discussed, $A(x)$ will be written $A_{\phi}[K](x)$ (for fixed $\phi$ ) or $A_{K}[\phi](x)$ (for fixed $K$ ). Thus, we seek to determine $f$ from knowledge of:

$$
\int_{B(x)} f d y=A(x)
$$

An inversion formula for this problem can be obtained by recognizing the integral in (3) as a convolution and applying the Fourier transform. If $\mu$ is Lebesgue measure concentrated on the unit ball $B(0)$.

$$
\int_{B(x)} f d y=\int_{B(0)} f(x-t) d \mu(t)=f * \mu .
$$

Application of the Fourier transofrm, solving for $\hat{f}$ and Fourier inversion yields:

$$
\hat{f} \hat{\mu}=\hat{A} \quad \text { and } \quad f=\left[\hat{A}(\hat{\mu})^{-1}\right]^{\vee}
$$

where $\wedge$ and $\vee$ indicate Fourier transform and inverse Fourier transform, respectively. In order to justify (5) it must be shown that $\hat{A}(\hat{\mu})^{-1}$ has a Fourier transform. For this we first calculate $\hat{\mu}$.

Lemma 1. Let

$$
J_{v}(x)=\sum_{k=0}^{\infty} \frac{(-1)^{k}\left(\frac{x}{2}\right)^{v+2 k}}{k ! \Gamma(v+k+1)}
$$

be the Bessel function of order $v$. If $\mu$ is Lebesgue measure on the unit ball at 0 and $x=r x^{\prime}$, $r>0, x^{\prime} \in S^{N-1}$ are polar coordinates, then

$$
\hat{\mu}(x)=r^{-N / 2} J_{N / 2}(2 \pi r)
$$

Proof:

$$
\hat{\mu}(x)=\int_{\mathbb{R}^{N}}^{e^{-2 \pi i x \cdot t}} d \mu(t)=\int_{0}^{1} \int_{S^{N-1}} e^{-2 \pi i r s x^{\prime} \cdot t^{\prime}} d t^{\prime} s^{N-1} d s
$$

where $x=x^{\prime} r, t=t^{\prime} s, x^{\prime}, t^{\prime} \in S^{N-1}, r>0$ and $0 \leq s \leq 1$. The inner integral can be evaluated by integrating on the parallel $L_{\theta}^{x}=\left\{t^{\prime} \in S^{N-1}: t^{\prime} \cdot x^{\prime}=\cos \theta\right\}$ and then on $0 \leq \theta<\pi$. Then, using the Poisson integral representation of the Bessel function $J_{(N-2) / 2}$ (Stein and Weiss [3], p. 154), the result is:

$$
\hat{\mu}(x)=\int_{0}^{1} 2 \pi(r s)^{-[N-2 / 2]} J_{(N-2) / 2}(2 \pi r s) s^{N-1} d s .
$$

The final result (6) is obtained from this via a simple change of variable and an integration formula (1) on p. 45 of Erdelyi et al. [4] for Bessel functions. 
Lemma 1 shows that $(\hat{\mu})^{-1}$ is singular on the zero set $Z$ of $J_{N / 2}(2 \pi r)$. But $Z$ has Lebesgue measure zero, and off $Z, A(\hat{\mu})^{-1}=\hat{f}$ which is $L^{1}$. Thus, $\hat{A}(\hat{\mu})^{-1}$ is almost everywhere equal to an integrable function and the Fourier inversion is justified. (This justification of (5) was communicated to the author by E. T. Quinto [5]. This help was critical and is gratefully acknowledged.)

We collect these facts in the following theorem:

THEOREM 1. If $K, \phi$ and $f$ are in $C_{c}^{\infty}\left(\mathbb{R}^{N}\right)$ and $K \Delta \phi+\nabla \phi \cdot \nabla K=f$ on $\mathbb{R}^{N}$, then

$$
f(x)=\int_{\mathbb{R}^{N}} e^{2 \pi i x \cdot y} \frac{|y|^{N / 2}}{J_{N / 2}(2 \pi|y|)} \hat{A}(y) d y,
$$

with

$$
A(x)=\int_{S(x)} K \frac{\partial \phi}{\partial n} d s=\int_{S(x)} K \sum_{i=1}^{N} \phi_{x_{i}} n_{i} d s .
$$

A glance at (7) shows that the inversion formula makes sense as long as $\hat{A}$ is defined and $\hat{A}(\hat{\mu})^{-1}$ is integrable. We now show that (7) constitutes a weak solution to the identification problem in the sense that (7) is meaningful for $K \phi_{x_{i}} \in L^{2}\left(\mathbb{R}^{N}\right)$ and that it satisfies the differential equation (1) when $\phi \in C^{2}\left(\mathbb{R}^{N}\right)$ and $K \in C^{1}\left(\mathbb{R}^{N}\right)$. This is somewhat surprising since only unit balls were involved in the derivation of (7).

THEOREM 2. The inversion formula (7) is a weak solution to the source identification problem.

Proof: Let $K \in C^{1}\left(\mathbb{R}^{N}\right), \phi \in C^{2}\left(\mathbb{R}^{N}\right)$. The mean $A(x)$ can be written as a convolution as follows:

$$
A(x)=\int_{S(x)} K \sum_{i=1}^{N} \phi_{x_{i}} n_{i} d s=\sum_{i=1}^{N} K \phi_{x_{i}} * \mu_{i}
$$

where $\mu_{i}=n_{i} d s$ on the sphere $S^{N-1}$. Taking Fourier transforms gives

$$
\hat{A}=\sum_{i=1}^{N} \widehat{K \phi}_{x_{i}} \hat{\mu}_{i} \text {. }
$$

The calculation of $\hat{\mu}_{i}$ is sketched in the next lemma.

LEMMA 2. If $\mu_{i}=n_{i} d s$ on the unit sphere $S^{N-1}$, then

$$
\hat{\mu}_{i}(x)=2 \pi i x_{i} r^{-N / 2} J_{N / 2}(2 \pi r) .
$$

Proof: Let $I(x)=\int_{S^{N-1}} e^{-2 \pi i r x \cdot t^{\prime}} d t^{\prime}$. By referring to the calculation of the inner integral in Lemma 1, differentiating I under the integral sign and using a differentiation formula ((51) on page 11 of Erdelyi et al. [4]) for Bessel functions, the result follows.

Now from Lemmas 1 and 2 and the expression of $\hat{A}$ given above, the inversion formula (7) becomes:

$$
\begin{aligned}
f(x) & =2 \pi i \int_{\mathbb{R}^{N}} e^{2 \pi i x \cdot y} \frac{|y|^{N / 2}}{J_{N / 2}(2 \pi|y|)} \sum_{i=1}^{N} x_{i} \frac{J_{N / 2}(2 \pi|y|)}{|y|^{N / 2}} \widehat{K \phi}_{x_{i}}(y) d y \\
& =2 \pi i\left(\sum_{i=1}^{N} x_{i}\left(\widehat{K \phi}_{x_{i}}\right)(x)\right)^{\vee} \\
& =\sum_{i=1}^{N} \frac{\partial}{\partial x_{i}}\left(\widehat{K \phi}_{x_{i}}\right)(x)=K(x) \Delta \phi(x)+\nabla K(x) \cdot \nabla \phi(x) .
\end{aligned}
$$


On the other hand, if $K \phi_{x_{i}} \in L^{2}\left(\mathbb{R}^{N}\right)$ the integral version of Minkowski's inequality (Stein [6], page 271) shows that $A=\sum_{i=1}^{N} K \phi_{x_{i}} * \mu_{i}$ is in $L^{2}$. The integral formula (7) is then meaningful via the $L^{2}$ theory of Fourier transforms.

The next theorem gives a continuous dependence on problem data for the source identification problem on $\mathbb{R}^{N}$. We seek to control the $L^{p}$ norm of $f$ with the $L^{p}$ norm of $K$ or $\phi$ for suitable $p$. It will be seen that the exponents $p$ for which this can be done depend upon the dimension $N$. The main ingredients here are $L^{p}$ estimates of spherical maximal functions and a Fourier multiplier theorem. The following two lemmas give these basics.

Lemma 3. (a) If $f$ is Lebesgue measurable on $\mathbb{R}^{N}$, then for almost all $x, f$ restricted to the sphere $S_{\alpha}(x)=\{y:|x-y|=\alpha\}$ is measurable with respect to Lebesgue measure on $S_{\alpha}(x)$, for all $\alpha>0$.

(b) Let $p>N /(N-1)$ with $N \geq 3$. If

$$
\left(M_{r} f\right)(x)=\int_{S^{N-1}} f(x-r y) d s(y), \quad(M f)(x)=\sup _{r>0}\left|\left(M_{r} f\right)(x)\right|
$$

then $\|M f\|_{p} \leq A_{p}\|f\|_{p}$ for a constant $A_{p}$ which is dependent on $N$.

Proof. See Theorem $A^{\prime}$ of Stein and Wainger [7].

Lemma 4. Suppose $m(x)$ is of class $C^{k}$ in the complement of the origin in $\mathbb{R}^{N}$, where $k>N / 2$. If for each multi-index $\alpha,\left|(\partial / \partial x)^{\alpha} m(x)\right| \leq B|x|^{-\alpha}$ with $B=$ constant, $|\alpha| \leq k$, then $\left\|(m \hat{h})^{\vee}\right\|_{p} \leq A_{p}\|h\|_{p}$ where the constant $A_{p}$ depends on $p, B$, and $N$.

Proof: This is Theorem 3 on page 96 of Stein [6]. The Sobolev spaces $W^{p, q}(\Omega)$ of all functions whose distributional derivatives of order up to and including $p$ are in $L^{q}$ will be needed in the following result which gives the continuous dependence of the source $f$ on the input data $K$ or $\phi$.

TheOREM 3. Assume $N \geq 3$ and $p>N /(N-1)$.

(a) For fixed $\phi$ which is bounded and $C^{1}$, the correspondence $K \rightarrow f$ given by (7) is continuous from $L^{p}\left(\mathbb{R}^{N}\right)$ to $L^{p}\left(\mathbb{R}^{N}\right)$ provided $f$ has an integrable Fourier transform.

(b) For fixed bounded and continuous $K$, the correspondence $\phi \rightarrow f$ given by (7) is continuous from $L^{p}\left(\mathbb{R}^{N}\right)$ to $L^{p}\left(\mathbb{R}^{N}\right)$ provided $\phi$ is in $W^{1, p}\left(\mathbb{R}^{N}\right)$ and $f$ has an integrable Fourier transform.

Proof: (a) It must be shown that the linear transformation $T \circ A_{\phi}$ given by $T\left(A_{\phi}(K)\right)=$ $\left[\widehat{A_{\phi}(K)}(\hat{\mu})^{-1}\right]^{\vee}$ is continuous on $L^{p} \cap\left(T \circ A_{\phi}\right)^{-1}\left\{f \in L^{p}: \hat{f} \in L^{1}\right\}$, where the means $A$ are defined by

$$
A_{\phi}(K)=A_{K}(\phi)=\int_{S(x)} K \frac{\partial \phi}{\partial n} d s .
$$

First it is shown that $A_{\phi}$ is continuous on $L^{p}$. For this we use Lemma 3 with $K \in L^{p}$ to get:

$$
\begin{aligned}
\left\|A_{\phi}(K)\right\|_{p} & =\left\|\int_{S(x)} \sum_{i=1}^{N} K \phi_{x_{i}} n_{i} d s\right\|_{p} \leq \sum_{i=1}^{N}\left\|\int_{S(x)}\left|K \phi_{x_{i}}\right| d s\right\|_{p} \\
& \leq \sum_{i=1}^{N} A_{i p}\left\|K \phi_{x_{i}}\right\|_{p}, \quad A_{i p}=\text { constant }
\end{aligned}
$$




$$
\leq N\left(\max _{1 \leqslant i \leqslant N} A_{i p}\right)\left(\sup _{\substack{1 \leqslant i \leqslant N \\ x \in \mathbb{R}^{N}}}\left|\phi_{x_{i}}(x)\right|\right)\|K\|_{p}
$$

Let $A_{\varepsilon}=\bar{B}_{1 / \varepsilon}(0) \cap\left(\cup_{i=1}^{\infty}\right) B_{\varepsilon}\left(j_{v, N / 2}\right)^{c}$, where $B_{r}(x)$ is the ball of radius $r$ about $x$ and $j_{v, N / 2}$ is the $v$ th zero of $J_{N / 2}(2 \pi|x|)$. Let $\chi_{\varepsilon}$ be a $C^{\infty}$ function which is 1 on $A_{\varepsilon}$ and with support contained in $A_{\varepsilon / 2}$. If $m_{\varepsilon}(x)=\left[J_{N / 2}(2 \pi|x|)(|x|)^{-N / 2}\right]^{-1} \chi_{\varepsilon}(x)$, then Lemma 4 implies that the map $T_{\varepsilon}: L^{2} \cap L^{p} \rightarrow L^{p}$ given by $T_{\varepsilon}(h)=\left(m_{\varepsilon} \hat{h}\right)^{\vee}$ has a continuous extension (also denoted by $T_{\varepsilon}$ ) to $L^{p}$. After extending $T_{\varepsilon}$ to $L^{p}$, the composition $T_{\varepsilon} \circ A_{\phi}$ is continuous on $L^{p}$. On the other hand, since $\hat{f} \in L^{1}$ and $\left|m_{\varepsilon}(x) \hat{A}(x)\right| \leq\left|(\hat{\mu})^{-1}(x) \hat{A}(x)\right| \in L^{1}$, the dominated convergence theorem gives: $\lim _{\varepsilon \rightarrow 0}\left(T_{\varepsilon} \circ A_{\phi}\right)(K)=f=\left(T \circ A_{\phi}\right)(K)$. The continuity of $T \circ A_{\phi}$ now follows from a corollary of the uniform boundedness principle. Assertion (b) is proved with the same argument with a slight and evident modification of (8).

Remarks: A notable omission from the statement of Theorem 3 is the case $N=2$. The result of Lemma 3 is false for $N=2$ and $p \leq N /(N-1)$. It is also false for $N=1$ and any $p<\infty$. The case $N=2, p>3 / 2$ is an open problem. For a discussion of these matters see Stein and Wainger [7].

If the assumption $\hat{f} \in L^{1}$ is dropped some "conditional" continuity can still be obtained. By this it is meant that if $K_{1}$ and $K_{2}$ correspond (for fixed $\phi$ ) to sources $f_{1}$ and $f_{2}$, and $K_{1}$ agrees exactly with $K_{2}$ to some extent and is $L^{p}$ close to $K_{2}$, then $f_{1}$ is $L^{p}$ close to $f_{2}$. For example, the following corollary is offered.

Corollary 1. Let $\varepsilon>0, p>N /(N-1)$ and $\phi$ be $C^{1}$ and bounded on $\mathbb{R}^{N}$. If $K_{1}$ and $K_{2}$ are in $L^{2} \cap L^{p}$ and $A_{\phi}\left(K_{1}-K_{2}\right)$ vanishes on the union of $\varepsilon$-balls about the zeros of $J_{N / 2}(2 \pi)$ $x \mid$ ) and off the $1 / \varepsilon$ ball about the origin, then there is a constant $C$ such that

$$
\left\|T \circ A_{\phi}\left(K_{1}-K_{2}\right)\right\|_{p} \leq C\left\|K_{1}-K_{2}\right\|_{p} .
$$

Proof: Using the notation of the theorem, $T=T_{\varepsilon}$ and the result follows.

An analogous corollary can also be stated for varying $\phi$ and fixed $K$.

Bounded domains. If $K \in C^{1}(\bar{D})$ and $\phi \in C^{2}(\bar{D})$, with $D$ a bounded domain in $\mathbb{R}^{N}$, the integral formula (7) can be used to determine $f$ if $K$ and $\phi$ are first extended to $\mathbb{R}^{N}$ in such a way that the above convolutions and transforms are meaningful. The extension can be made by multiplying $C^{2}$ extensions of $K$ and $\phi$ by a $C^{\infty}$ function of compact support which has value 1 on $\bar{D}$. The continuous dependence of Theorem 3 then holds for the extended functions. For example (using the same symbols for the extended functions), if $K_{1}$ is a perturbation of $K$ with $K_{1} \in L^{p}, p>N /(N-1)$, and $\left\|K-K_{1}\right\|_{p}$ is small, then $\left\|f_{1}-f\right\|_{p}$ is small, where $f_{1}$ is the source resulting from the use of (7) with $K_{1}$ and $\phi$. This result is useful if $K$ is thought of as extended from the "true" permeability on $D$ and $K_{1}$ is extended from nonsmooth measured data on $D$.

3. A stochastic source identification problem. It is now assumed that $K, \phi$, and $f$ are random functions defined on $\mathbb{R}^{N} \times \Omega$ where $(\Omega, F, P)$ is a probability space. We also suppose:

(a) $K \phi_{x_{i}} \in L^{1} \cap L^{2}$ with respect to the measure space $\left(\mathbb{R}^{N} \times \Omega, \mathscr{L} \otimes F, m \otimes P\right)$ where $\mathscr{L}$ is the $\sigma$-algebra of Lebesgue-measurable sets and $m$ is Lebesgue measure. 
Under assumption (a), Fubini's theorem and Theorem 2 says that for each $\omega \in \Omega$,

$$
f(x, \omega)=\int e^{2 \pi i x \cdot y} \frac{|y|^{N / 2}}{J_{N / 2}(2 \pi|y|)} A\left[\phi(\cdot, \omega), K(\cdot, \omega)^{\wedge}(y) d y\right.
$$

is a weak solution to the source identification problem on $\mathbb{R}^{N}$. It is of interest to calculate the expected value and variance of $f(x, \cdot)$ as a function of $x$ in terms of statistical information about the problem data. The calculation of $E(f(x, \cdot))=\int f(x, \omega) d P(\omega)$ turns out to be essentially the same as in the deterministic problem with a covariance function replacing $K \phi_{x_{i}}$. This can be seen by simply interchanging expectation with all the integrals occurring in (9). After justifying these interchanges, the methods of the previous section can be used to estimate $\|E f\|_{p}$.

THEOREM 4. Let $K \phi_{x_{i}}$ satisfy assumption (a). If $f(x, \omega)$ is the solution of the random source identification problem given in (9), then:

$$
(E f)(x)=\left[\frac{|y|^{N / 2}}{J_{N / 2}(2 \pi|y|)} A\left[E\left(K \frac{\partial \phi}{\partial n}\right)^{\wedge}(y)\right]^{\vee}(x)\right],
$$

where

$$
A[h](t) \stackrel{\text { def }}{=} \int_{S(t)} h d s, \quad E\left(K \frac{\partial \phi}{\partial n}\right) \stackrel{\text { def }}{=} E\left(\sum_{i} K \phi_{x_{i}}\right) n_{i} .
$$
that:

If $\hat{f}(y, \omega) \in L^{1}\left(\mathbb{R}^{N}\right)$ for each $\omega \in \Omega$, then for $p>N /(N-1)$ there is a constant $D_{p}$ such

$$
\|E f\|_{p} \leq D_{p}\left\|E\left(K \frac{\partial \phi}{\partial n}\right)\right\|_{p} .
$$

The proof of Theorem 4 requires the following lemma:

LEMMA 4. For almost all $t$,

$$
\int d p(\omega) \int_{0}^{2 \pi} K \frac{\partial \phi}{\partial n}\left(t+e^{i \theta}, \omega\right) d \theta=\int_{0}^{2 \pi} E\left(K \frac{\partial \phi}{\partial n}\right)\left(t+e^{i \theta}\right) d \theta
$$

Proof: Lemma 3(a) shows that for fixed $\omega \in \Omega$, the integral $\int K \phi_{x_{i}}(x-y, \omega) d \mu_{i}(y)$ with $d \mu_{i}=n_{i} d s$ is well defined on $\mathbb{R}^{N}-\mathcal{N}$ where $\mathcal{N}$ has Lebesgue measure zero. On the other hand,

$$
\iint\left|K \phi_{x_{i}}(x-y, \omega)\right| d x d \mu_{i}(x) \leq\left\|K \phi_{x_{i}}\right\|_{1}(\omega) \cdot\left|\mu_{i}\right|(S(0))
$$

Hence, Fubini's theorem implies that:

$$
\iint_{\mathbb{R}^{N}-\mathcal{N}} K \phi_{x_{i}}(x-y, \omega) d \mu_{i}(y) d x<\infty, \quad \int K \phi_{x_{i}}(x-y, \omega) d \mu_{i}(y)<\infty
$$

almost everywhere. Another application of Fubini's theorem gives:

$$
\int d P(\omega) \int_{0}^{2 \pi} K \frac{\partial \phi}{\partial n}\left(t+e^{i \theta}, \omega\right) d \theta=\int d P(\omega) \int \sum_{i=1}^{N} K \phi_{x_{i}}(t-s, \omega) d \mu_{i}(s)
$$




$$
\begin{aligned}
& =\int \sum_{i=1}^{N} E\left(K \phi_{x_{i}}\right)(t-s) d \mu_{i}(s) \\
& =\int_{0}^{2 \pi} E\left(K \frac{\partial \phi}{\partial n}\right)\left(t+e^{i \theta}\right) d \theta .
\end{aligned}
$$

This finishes Lemma 4. Applying Lemma 4 and Fubini's theorem to take expectations inside the Fourier transform integrations yields (10). The method of proof of Theorem 3 with $K(\partial \phi / \partial n)$ replaced by $E(K(\partial \phi / \partial n)$ gives (11). This completes the proof of Theorem 4 .

Next we calculate an upper bound for the $L^{1}$ norm of the variance of $f$ in terms of known data.

THEOREM 5. If $N /(N-1)>2$, then, using the notation of Theorem 4 , the variance of $f$ (as a function on $\mathbb{R}^{N}$ ) satisfies the estimate

$$
\|\operatorname{Var}(f)\|_{1} \leq D_{2}^{2}\left\|E\left(K \frac{\partial \phi}{\partial n}\right)\right\|_{2}^{2}+C E\left(\|K\|_{2}^{2}\right)
$$

for a constant $C$.

Proof: We may assume $E[K(\partial \phi / \partial n)] \in L^{2}$; otherwise (13) is trivially satisfied. Since $\operatorname{Var}(f)=E\left(f^{2}\right)-(E(f))^{2}$ it follows that

$$
\|\operatorname{Var}(f)\|_{1} \leq\left\|E\left(f^{2}\right)\right\|_{1}+\left\|(E f)^{2}\right\|_{1} .
$$

Schwarz's inequality, (11) and Theorem 4 imply that

$$
\left\|(E f)^{2}\right\|_{1} \leq D_{2}^{2}\left\|E\left(K \frac{\partial \phi}{\partial n}\right)\right\|_{2}^{2} .
$$

As for the first summand of (14), assumption (a) and Fubini's theorem show

$$
\left\|E\left(f^{2}\right)\right\|_{1}=E\left\|f^{2}\right\|_{1}=E\|f\|_{2}^{2} .
$$

Since $f(x, \omega), K(x, \omega), \phi(x, \omega)$ satisfy (9) for each fixed $\omega \in \Omega$, Theorem 3 provides a constant $C$ such that

$$
\|f\|_{2}^{2}(\omega) \leq C\|K\|_{2}^{2}(\omega) \quad \text { for all } \omega \in \Omega
$$

Consequently,

$$
\left\|E\left(f^{2}\right)\right\|_{1} \leq E\left(C\|K\|_{2}^{2}\right)=C E\left(\|K\|_{2}^{2}\right) .
$$

Thus the estimate (13) follows from (16) and (15). This finishes Theorem 5.

Because of the linearity of (9), the above two theorems can be thought of as continuous dependence results.

If the stochastic source identification problem is posed on a bounded domain in $D \subset \mathbb{R}^{N}$, results analogous to Theorems 4 and 5 can still be established. Suppose $K(x, \omega) \in C^{1}(\bar{D}), \phi(x, \omega) \in C^{2}(\bar{D})$ for each $\omega \in \Omega$. Then each $\omega$-section of $K$ and $\phi$ can be extended to $\mathbb{R}^{N}$ as previously done. After the extensions are made, an integral formula analogous to (9) (with $K$ and $\phi$ replaced by their extensions) solves the random source identification problem on $\mathbb{R}^{N}$ and in particular, on $D$. There are continuous dependence estimates for $E f$ and $\operatorname{Var}(f)$ corresponding to (11) and (13) for the extended functions. 


\section{REFERENCES}

[1.] J. R. Cannon and P. DuChateau, An inverse problem for an unknown source in a heat equation, J. Math. Anal. Appl. 75, 465-485 (1980)

[2.] A. N. Tikhonov, Solutions to ill-posed problems, Winston-Wiley, New York, 1978

[3.] E. M. Stein and G. Weiss, Introduction to Fourier analysis on Euclidean spaces, Princeton University Press, 1971

[4.] A. Erdelyi, ed., Higher transcendental functions, Vol. II, McGraw-Hill, 1953

[5.] B. T. Quinto, personal communication.

[6.] E. M. Stein, Singular integrals and the differentiability properties of functions, Princeton University Press, 1970

[7.] E. M. Stein and S. Wainger, Problems in harmonic analysis related to curvature, Bull. AMS 84, 1239-1295 (1978) 